CERN-TH/2000-146

\title{
THE SO-CALLED RENORMALIZATION GROUP METHOD APPLIED TO THE SPECIFIC PRIME NUMBERS LOGARITHMIC DECREASE
}

\author{
A. Petermann \\ TH Division, CERN \\ CH - 1211 Geneva 23
}

\begin{abstract}
A so-called Renormalization Group ( $\mathrm{RG}$ ) analysis is performed in order to shed some light on why the density of prime numbers in $\mathbb{N}^{*}$ decreases like the single power of the inverse neperian logarithm.
\end{abstract}

CERN-TH/2000-146

May 2000 


\section{Part I}

\section{The most elementary proof of the Prime Num- bers Theorem}

These few lines are not part of the proof. They simply show the history which has led to the starting point of our proof. The two main steps of it involve only formal elementary algebra, with no recourse naturally to Functions' theory nor complex variables. Euler proved in 1747 [1], quite formally, that the prime numbers are linked with natural integers, by establishing what is universally known as "Euler identity" and which is so famous that we do not recall it here.

From this identity, one can deduce straight forwardly an approximate formula円 giving

$$
\text { (I) } \sum_{p<\Lambda} 1 / p=1 \cdot \log (\log \Lambda)+\cdots
$$

This result has been refined, mainly by Mertens [2] with the aim to establish the value of constants possibly entering a much more exact expression of the sum in (I). This expression, we call it "Euler-Mertens identity" and is the starting point of our proof, the formula [ of our theorem.

\section{$\underline{\text { Proof }}$}

Consider the Euler-Mertens identity (I), exact when $\Lambda \rightarrow \infty$. We introduce it under the following form (1) and apply the RG analysis [3]

$$
\bar{F}=(\log \log \Lambda)^{1} \int^{\Lambda} \frac{d n \bar{n}(n)}{n} \cong C^{t e} \cong 1, \Lambda \rightarrow \infty
$$

$\bar{n}(n) d n$ is a measure $d f(n)$ of the Stieltjes type and $\bar{n}(n)$ can be considered as a density in the physical sense?.

Then, since

$$
\Lambda \partial / \partial \Lambda \bar{F}=\frac{\partial}{\partial \log \Lambda}(\log \log \Lambda)^{-1} \cdot \int^{\Lambda} \frac{d n \bar{n}(n)}{n}+\frac{\bar{n}(\Lambda)}{\log \log \Lambda}
$$

\footnotetext{
${ }^{1}$ Which, in fact, is the strict equivalent of Euler identities.

${ }^{2} d f(n)=\bar{n}(n) d n$ and, according to the result (3) $f(\Lambda)=\int{ }^{\Lambda} \frac{d n}{\log n} \cong \operatorname{Li}(\Lambda)$.
} 


$$
=-(\log \Lambda)^{-1}(\log \log \Lambda)^{-2} \cdot \int^{\Lambda} \frac{d n \bar{n}(n)}{n}+\frac{\bar{n}(\Lambda)}{\log \log \Lambda}
$$

one deduces, by the RG method, (since $d \bar{F} / d \log \Lambda=0$ )

$$
\begin{aligned}
0 & =-\frac{(\log \Lambda)^{-1}}{\log \log \Lambda} \cdot F+\frac{\bar{n}(\Lambda)}{\log \log \Lambda}+\left[\frac{\partial \bar{n}(\Lambda)}{\partial \log \Lambda}\right] \frac{\delta}{\delta \bar{n}(\Lambda)} \bar{F} \\
& =\left[-(\log \Lambda)^{-1}+\bar{n}(\Lambda)\right](\log \log \Lambda)^{-1}+0\left(\frac{1}{\Lambda \cdot \log ^{2} \Lambda \cdot \log \log \Lambda}\right)
\end{aligned}
$$

So from 2, at this approximation one gets

$$
\bar{n}(\Lambda) \cong(\log \Lambda)^{-1}, \quad \Lambda \gg 1
$$

which is the prime Numbers theorem, since the density

$$
\bar{n}(\Lambda)=\Lambda^{-1} \cdot \pi(\Lambda)
$$

\section{Part II}

\section{The RG Equation for the Density of Prime Numbers}

The density of natural integers is scale invariant:

$$
\lambda \frac{\partial}{\partial \lambda} \bar{d}\left(n_{i} \lambda, \bar{d}(1)\right)=0
$$

$\bar{d}$ being the density around $n_{i} \lambda$, and $\lambda \frac{\partial}{\partial \lambda}$ the generator of scale transformations of the natural integers.

If, on the other hand, $n_{i}$ is a prime and $\lambda n_{i}$ is around another prime, say $n_{i}^{\prime}$, then

$$
\bar{d}\left(n_{i}^{\prime} \cong \lambda n_{i}, \bar{d}(1)\right) \neq \bar{d}\left(n_{i}, \bar{d}(1)\right) .
$$

\footnotetext{
${ }^{3}$ The RG-method has been designed in order to know how the structure of a theory gets modified when the scale is changed.
} 
In such a case, instead of having an equation like (4), expressing the invariance for scale changes, one uses, as is customary, the so-called Renormalization Group ( $\mathrm{RG}$ ) equation (or better: renormalization transformation equation) which generally substitutes Eq. (4) when scale invariance is broken. The strategy is to compensate the broken invariance, for example in the present case, by a density $\bar{d}$, which this time depends upon $\lambda$ and is different from that of (4), namely $\bar{d}(1)$. The RG equation, as is well known [3], reads

$$
\lambda \frac{\partial}{\partial \lambda} \bar{d}\left(n_{i} \lambda, \bar{d}(\lambda)\right)+\left[\frac{\lambda \partial}{\partial \lambda} \bar{d}(\lambda)\right] \frac{\partial}{\partial \bar{d}(\lambda)} \bar{d}\left(n_{i} \lambda, \bar{d}(\lambda)\right)=0
$$

Equation (5) fintroduces the quantity $\left[\lambda \frac{\partial}{\partial \lambda} \bar{d}(\lambda)\right] \frac{\partial}{\partial \bar{d}(\lambda)}$ which is a one-dimensional vector field $\underline{V}(\bar{d}(\lambda))$ on the axis of integer numbers.

Now, the problem is to solve (5) for $\bar{d}$.

This solution, as will be explained in the Appendix, when taken between two different numbers $N_{1}$ and $N_{2}$, turns out to be

$$
\frac{1}{\bar{d}\left(N_{1}\right)}-\frac{1}{\bar{d}\left(N_{2}\right)}=\log \frac{N_{1}}{N_{2}} .
$$

But, perhaps, more instructively for the scope of this short note,

$$
\bar{d}\left(t, \bar{d}\left(0, \bar{d}_{0}\right)=\frac{\bar{d}\left(0, \bar{d}_{0}\right)}{1+t \cdot \bar{d}\left(0, \bar{d}_{0}\right)}\right.
$$

with $t=\log N$, exhibiting the neperian logarithmic single power decrease as having its origin in the violation of the scale invariance symmetry.

Indeed, if in the region of large primes (say between $10^{15}$ and $2 \times 10^{17}$ ), the numerical results obtained by the use of (6), formula (6) does not tell else that, for each number $N_{1}$ and $N_{2}$,

$$
\frac{N}{\pi(N)}=\log N
$$

which is a 100 years-old, over-demonstrated asymptotic result [4, 5].

However, in this note, our aim is to look for the deep reason why the density of primes decreases with the single power of the natural logarithm.

\footnotetext{
${ }^{4} n_{i} \lambda \cong$ prime, as $n_{i}$ is.
} 
We hope that we have been able to shed some light on this fact: the breaking of a symmetry, namely that of scale invariance with generator $\lambda \frac{\partial}{\partial \lambda}$, is the very factor responsible for this specific decrease.

The coincidence of the results obtained is striking when compared to the formulas of the first non-trivial approximation of Quantum ChromoDynamics (mutatis mutandis, of course, the concepts between two such different fields).

But a main common feature emerges: in both cases the two fields are afflicted by the same broken symmetry, that of scale invariance. 


\section{APPENDIX}

1) For natural integers, scale invariance holds for the density $\bar{d}(n)$, i.e. when $n \rightarrow \lambda n, \bar{d}(n)=\bar{d}(\lambda n)$.

2) For primes $p_{i}$,

$$
p_{i} \rightarrow \lambda p_{i} \cong p_{j}, \bar{d}\left(p_{j}\right) \neq \bar{d}\left(p_{i}\right),
$$

so that $\bar{d}$ becomes a function of $\lambda$.

One finds easily that a functional equation of the type

$$
\bar{d}\left(\lambda n_{i}, \bar{d}(\lambda)\right)=\bar{d}\left(n_{i}, \bar{d}_{0}\right)
$$

( $\bar{d}_{0}$ fixed, and $n_{i}$ representing primes as well as $\lambda n_{i}$ in the dose vicinity of $n_{j}$, prime itself) exists.

The RHS is $\lambda$-independent and one gets at once

$$
\lambda \frac{\partial}{\partial \lambda} \bar{d}\left(\lambda n_{i} \cong n_{j}, \bar{d}(\lambda)\right)+\left[\lambda \frac{\partial}{\partial \lambda} \bar{d}(\lambda)\right] \frac{\partial}{\partial \bar{d}(\lambda)} \bar{d}\left(\lambda n_{i}, \bar{d}(\lambda)\right)=0 .
$$

Or else, calling $t=\log \lambda$ and passing to logarithmic variables

$$
\left[\frac{\partial}{\partial t}+\underline{V}(\bar{d}(t)] \bar{d}\left(t+\log n_{i}, d(t)\right)=0\right.
$$

with

$$
\underline{V}(\bar{d}(t))=\left[\frac{\partial}{\partial t} \bar{d}(t)\right] \frac{\partial}{\partial \bar{d}(t)} .
$$

To solve (A.2), one proceeds in the following way

$$
\begin{aligned}
\bar{d}\left(\delta t, \bar{d}\left(0, \bar{d}_{0}\right)\right)= & \bar{d}\left(0, \bar{d}\left(0, \bar{d}_{0}\right)\right. \\
& +\delta t \underline{V}\left(\bar{d}\left(0, \bar{d}_{0}\right)\right) \cdot \bar{d}\left(0, \bar{d}_{0}\right)+0\left(\delta t^{2}\right)
\end{aligned}
$$

by Taylor-expanding $\bar{d}$ around $\delta t=0$.

According to the properties of flows of vector fields, one has $\bar{d}(0, x)=x$, that is, for example, $\bar{d}\left(0, \bar{d}\left(0, \bar{d}_{0}\right)\right)=\bar{d}\left(0, \bar{d}_{0}\right)=\bar{d}_{0}, \bar{d}_{0}$ being a fixed arbitrary density. 
One gets then

$$
\bar{d}\left(\delta t, \bar{d}_{0}\right)-\bar{d}_{0}=\delta t \underline{V}\left(\bar{d}_{0}\right) \cdot \bar{d}_{0}
$$

or周

$$
\bar{d}\left(\delta t, \bar{d}_{0}\right)=\left(1+\delta t \underline{V}\left(\bar{d}_{0}\right)\right) \bar{d}_{0}
$$

By theorems by Chebyshev and Mertens [6, 2], $V(x)$ can be shown to be quadratic in its argument $x$.

It remains to exponentiate the RHS of (A.4):

$$
\begin{aligned}
\bar{d}\left(t, \bar{d}_{0}\right) & =\left(1+t \underline{V}\left(\bar{d}_{0}\right) \frac{\partial}{\partial \bar{d}_{0}}+\frac{t^{2}}{2 !} V\left(\bar{d}_{0}\right) \frac{\partial}{\partial \bar{d}_{0}} \cdot V\left(\bar{d}_{0}\right) \frac{\partial}{\partial \bar{d}_{0}}+\cdots\right) \bar{d}_{0} \\
& =\frac{\bar{d}_{0}}{1+t \bar{d}_{0}} ; \quad\left(V\left(\bar{d}_{0}\right)=-\bar{d}_{0}^{2} \cdot \text { see above }\right)
\end{aligned}
$$

(A.5) is the formula (7) of the text and seems to us to be an explanation we were searching for, to explain the decrease of $\bar{d}\left(t, d_{0}\right)$ with a single power of the natural $\operatorname{logarithm} t=\log N$ (Remember that $\bar{d}(t, \cdots) \equiv \bar{d}\left(e^{t}, \cdots\right)$, as (A.2) and (A.3) show without further comments.)

As a final remark, (6) follows straight forwardly from (7) by trivial algebra.

Take (7) with two different values for $t: t_{1}=\log N_{1}$, and $t_{2}=\log N_{2}$. It follows at once that

$$
\bar{d}^{-1}\left(t_{1}, \bar{d}_{0}\right)-\bar{d}^{-1}\left(t_{2}, \bar{d}_{0}\right)=\log N_{1} / N_{2}
$$

(Additionally it confirms the arbitrariness of $\bar{d}_{0}$ which might be chosen at will; $\bar{d}_{0}=1$, for example.)

\footnotetext{
${ }^{5}$ In passing we recall a well-known property of the flows $\bar{d}(t, x)$; namely they satisfy the one-parameter Abelian group: $\bar{d}(s+t, x)=\bar{d}(t, \bar{d}(s, x))$, i.e. the composition law $\bar{d}_{s+t}=$ $\bar{d}_{t} \circ \bar{d}_{s}$. This group is trivially generated as the one-parameter group of diffeomorphisms by the vector field $V$ on the manifold considered. For details see Ref. 33a.)
} 


\section{References}

[1] L. Euler, "Varia observationes circa series infinitas", Comment. Acad. Sci. Imp. Petropol. 9, 160-188 (1744-1747).

[2] F. Mertens, "Ein Beitrag zur analytischen Zahlentheorie"; J. reine angew. Math. 78 (1874) 46-62.

[3] E.C.G. Stueckelberg, A. Petermann, Helv. Phys. Acta , 26 (1953) 499. See: a) A.Petermann, CERN-TH/99-389, hep-th.9912-131, for a modern view on the subject.

[4] J. Hadamard, "Sur la distribution des zéros de la fonction $\zeta(s)$ et ses conséquences arithméthiques", Bull. Soc. Math. France 24 (1896) 199220;

Ch. De La Vallée Poussin, "Recherches analytiques sur la théorie des nombres premiers", Ann. Soc. Sci. Bruxelles 20 (1896) 183-256 et 281297.

[5] A. Selberg, "An elementary proof of the prime number theorem", Ann. Of Math. (2) 50 (1949) 305-313.;

P. Erdös, "On a new method in elementary number theory which leads to an elementary proof of the prime number theorem", Proc. Nat. Acad. Sci. USA 35 (1949) 374-384;

Then follow many other proofs using a refinement of the same method, each with a slight simplification.

[6] P.L. Chebychev, "Theory of congruences", St Petersburg (1849), "Mémoires présentées à l'Académie Impériale des Sciences de St Petersbourg par divers membres" 7 (1850-1854) 15-33; and J. Math. (1) 17 (1852) (366-390). 